\title{
UN-SENSORED VERY HIGH RESOLUTION LAND-COVER MAPPING
}

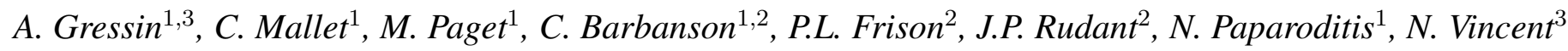 \\ ${ }^{1}$ IGN/SRIG, MATIS; ${ }^{2}$ UPEM; ${ }^{3}$ Paris-Descartes University, LIPADE - SIP
}

\begin{abstract}
Index Terms - Remote sensing, change detection, land cover, satellite imagery.
\end{abstract}

\section{INTRODUCTION}

Land-Cover geodatabases (LC-DB) are inevitable inputs in various challenging domains, linked to environmental monitoring or public policy planning. In recent years, the users' needs have tremendously increased, both in terms of accuracy (semantically and geometrically), exhaustiveness and updateness. Therefore, automatic methods have been developed in the literature in order to update or refine such LC-DB. Moreover, methodological developments have been accompanied with a higher availability of geospatial sensors and remote sensing (RS) data, through national or international infrastructures and image archives: in 2014, more than 80 civilian Earth observation missions are active and 12 satellites are launched every year [1]. Such recent sensors allow to acquire data with increasing spectral, spatial and temporal resolutions. Aerial remote sensing data, whether optical or LIDAR, is following the same trend. Consequently, today, we face a high number of sensor types and configurations, providing complementary information (spectral and textural information for optical data, height and geometric information for LIDAR data, amplitude, phase for SAR sensors and even polarimetry) for the characterization of the objects of the Earth surface and for their semantization into land-cover classes. In this context, many studies have shown that the fusion of such various RS data allow to sharply improve landcover classifications, and a large part of recent literature has dealt with this subject [2-4]. However, it often remains limited to two or three sensors (or data types in case of optical images at various spatial resolutions), with a prevalence of one sensor over the other ones (mainly in terms of feature extraction).

In this paper, we present an improvement of an existing LCDB updating framework [5], by introducing a new data fusion step, which allows to deal simulaneously with such various available datasets. They are: airborne LIDAR point clouds, polarimetric SAR images and optical images. Three different decision-fusion methods are presented and compared over an area of interest mixing urban and natural landscapes and covered by the French national reference LC-DB.

\section{METHODOLOGY}

A hierarchical classification staking framework designed to update land-cover geodatabses has been introduced in [5]. This framework is decomposed into three main levels which are: (1) Training pixel selection for each $2 \mathrm{D}$ object of the DB of each class, leading to one classification of the full area of interest per DB object, (2) Fusion of the classifications obtained for each object of a class, at the class level, and (3) Final label assignment by merging the decisions of all the classes of interest.

In this paper, we focus on the second step, namely, class-level classification fusion. Here, the main idea is to learn the appearance of each object of the DB for each RS dataset, and then to merge those classifications. Moreover, initial SVM classifier has been replaced by a Random Forest (RF) method, allowing to deal with large feature set and to speed up computational time. For that purpose, we have first adapted the general framework, by computing specifically a large number of features for each RS data source. Then, a per-class selection step is performed on each RS dataset, resulting in one feature subset for each DB class and each RS dataset. Eventually, one "one-versus-all" classification per object is performed on each feature subset of the current object class's. This allows to 
retrieve for each RS dataset and each object of each DB class, a confidence map that describes the probability of a pixel to belong to the same class as the one of the object. The subsequent step consists in merging all object-level confidence maps of one class into a unique class-level confidence map (considered as the probability of each pixel to belong to the current class).

Then, three fusion methods are proposed and detailed below. First, the class-level confidence map is generated by computing the mean value of object-level confidence maps of the same class (Eq. 1). This method allows to reduce the effect of inconsistent classifications, but will discard low represented appearance of one heterogeneous class.

The second proposed method takes into account the correctness of the classification regarding the initial DB, by weighting each classification by its associated F-Score (Eq. 2).

$$
\begin{aligned}
\mathcal{P}_{\text {Mean }}^{C} & =\frac{1}{\sharp C} \sum_{O \in C} \mathcal{P}_{O}, \\
\mathcal{P}_{\text {F-Score }}^{C} & =\frac{1}{\sum_{O \in C} f_{O}} \sum_{O \in C} f_{O} * \mathcal{P}_{O} .
\end{aligned}
$$

where $\sharp C$ is the number of objects in the class $C, \mathcal{P}_{O}$ is the confidence map obtain with the object $O$ and $f_{O}$ is the FScore of the classification of object's class, by this object.

Finally, we propose to model the fusion process as a linear combination issue:

$$
P(p \in C)=\sum_{O \in C} a_{O} * \mathcal{P}_{O}(p)
$$

where, $P(p \in C)$ is the probability $P$ of the pixel $p$ to belong to the class $C$. This value is given by the initial DB as a binary value ( 1 if $p$ belongs to the class $C, 0$ otherwise). This overdetermined system can be solved with leastsquares method. However, this method can give negative coefficients, which have no meaning in terms of probability. Consequently, a stacked regression method, least-squares under non-negativity constrains (NNLS) [6], is introduced to determine the $a_{O}$ coefficients.

\section{DATASETS}

An area of interest near the city of Tarbes (Southern France) has been selected for the availability of both LC-DB and a large number of RS data sources (described below), and for its geographic configuration: a peri-urban area covered by forest, grassland, and different urban landscapes (residential, industrial, ... ). In this paper, a simplified LC DB is derived from the French National LC DB, composed of 23 classes. The 5 main classes have been extracted of the initial LC-DB, namely building area, road and parking lots, water, forest and grassland. These are the classes of interest in our experiments.

Remote sensing dataset are:

- One optical satellite image (SPOT 6);

- One airborne LIDAR point cloud composed of more than 10 million $3 \mathrm{D}$ points;

- and one TerraSAR-X image.

Details of each dataset are provided in Table 3. In particular, we assume that LC-DB and all RS datasets are correctly registrated, even if the proposed framework is able to deal with registration issues in case of low planimetric discrepancies.

\section{RESULTS}

First, on-purpose features are derived from each RS dataset: spectral, textural, and geometrical feature from optical image; features based on echo, heigh, local geometry and intensity information from LIDAR data; intensity from SAR data. LIDAR features are computed either at point level or using a local neighbourhood. In both cases, features are then rasterized (i.e.,, projected on a $2 \mathrm{D}$ grid at $1 \mathrm{~m}$ resolution). Those features have been individually assessed for LC-DB class discrimination, using variables importance from Random Forest. Preliminary results tend to show the predominance of optical sensor based features for vegetation classes (forest and grassland), whereas LIDAR features outperform the latter on anthropic classes (especially on dense urban landscapes). This is due the fact that they provide reliable and accurate height and geometric information for off-ground objects.

LIDAR and SAR data will be integrated with optical images, in various scenarii. Moreover, existing LC-DB will allow to perform a fine quality assessment of each scenario. 


\begin{tabular}{c|c|c|l} 
Name & Type & Date & Characteristics \\
\hline SPOT 6 & optical & April 2013 & $\begin{array}{l}1.5 \mathrm{~m} \text { in panchromatic and } 6 \mathrm{~m} \text { in color (red, green, blue and } \\
\text { near infrared) }\end{array}$ \\
$\begin{array}{c}\text { Optech 3100 } \\
\text { TerraSar-X }\end{array}$ & $\begin{array}{c}\text { LIDAR } \\
\text { SAR }\end{array}$ & $\begin{array}{l}\text { January 2013 } \\
\text { August 2014 }\end{array}$ & $\begin{array}{l}4 \mathrm{pts} \mathrm{m}^{2}, 10 \mathrm{million} \text { 3D points. } \\
\text { Band } \mathrm{X}(0.03 \mathrm{~m}), \text { Polarizations } \mathrm{HH} / \mathrm{VV} \text {, Ground range / azimuth } \\
\text { pixel size }: 1.7 \mathrm{~m} / 3.2 \mathrm{~m} .\end{array}$
\end{tabular}

Table 1. Remote sensing datasets description.
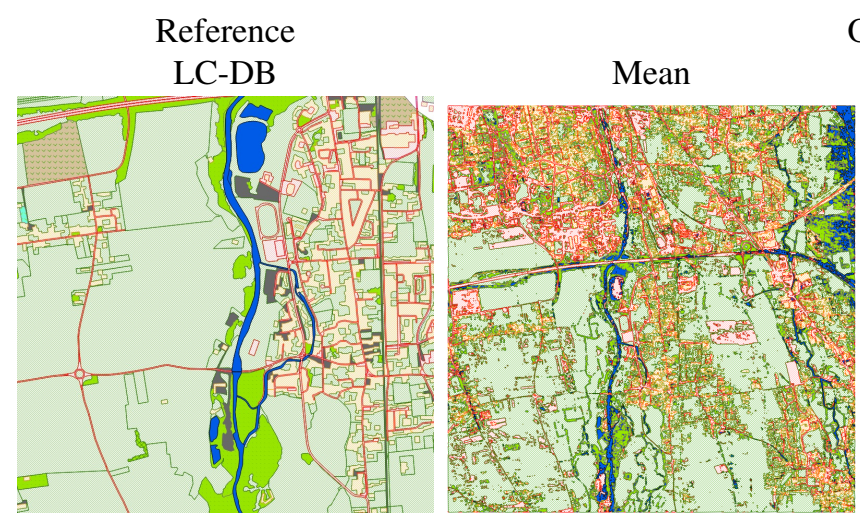

Object level classification fusion
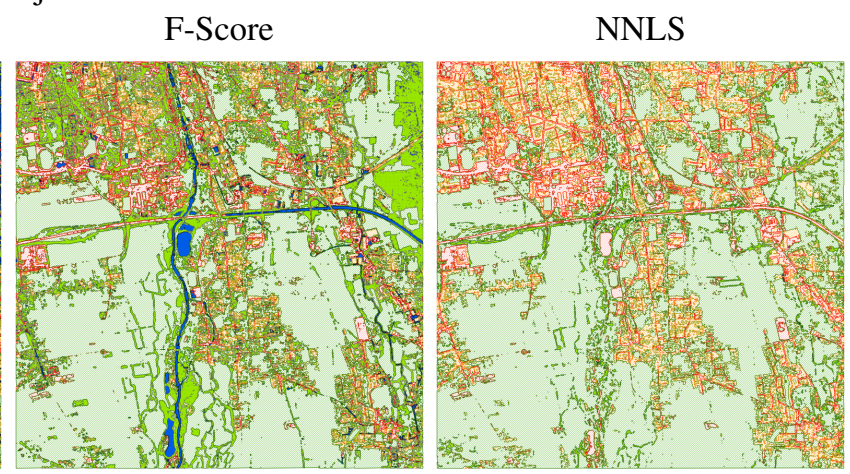

Table 2. Comparison of the 3 fusion results (Mean, F-Score, and NNLS), on 3 RapidEye images.

\begin{tabular}{|c|c|c|c|c|c|c|c|c|c|c|c|c|c|c|c|c|c|c|c|c|c|}
\hline & \multicolumn{3}{|c|}{ SP6 } & \multicolumn{3}{|c|}{$\mathrm{Li}$} & \multicolumn{3}{|c|}{ RA } & \multicolumn{3}{|c|}{ SP6-Li } & \multicolumn{3}{|c|}{ SP6-RA } & \multicolumn{3}{|c|}{ SP6-Li-RA } & \multicolumn{3}{|c|}{ Li-RA } \\
\hline & M & $\mathrm{F}$ & $\mathrm{N}$ & $\mathrm{M}$ & $\mathrm{F}$ & $\mathrm{N}$ & M & $\mathrm{F}$ & $\mathrm{N}$ & $\mathrm{M}$ & $\mathrm{F}$ & $\mathrm{N}$ & M & $\mathrm{F}$ & $\mathrm{N}$ & & $\mathrm{F}$ & $\mathrm{N}$ & M & $\mathrm{F}$ & $\mathrm{N}$ \\
\hline & \multicolumn{21}{|c|}{ Overall accuracy } \\
\hline & 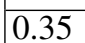 & 3 & 05 & 67 & 71 & 07 & 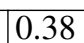 & & 0.63 & & & & 46 & & & .6 & & & .6 & 72 & $\overline{0 .}$ \\
\hline & \multicolumn{21}{|c|}{ Recall by class } \\
\hline building & 54 & 0.51 & 0.47 & 0.42 & 0.58 & 0.68 & .24 & 0.18 & 0.30 & 0.59 & 0 & 0.74 & 0.43 & 0.30 & 0.62 & 0.56 & 0.43 & 0.73 & 0.42 & 0.45 & 0.69 \\
\hline 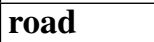 & 65 & 0 & 0. & 0.55 & 0. & 0.67 & 0.31 & 0.24 & 0.25 & 0.63 & 0.51 & 0.65 & 0.58 & 0.31 & 0.36 & 0.63 & U & 0.61 & 0.54 & 0.47 & 0.61 \\
\hline wate & 0.21 & 0.27 & 0. & 0.75 & 0.74 & 0.03 & 0.39 & 0.27 & 0.00 & 0.52 & 0.75 & 0.63 & 0.14 & 0.40 & 0.00 & 0.58 & 0.76 & 0.02 & 0.79 & 0.75 & 0.01 \\
\hline rest & 0.45 & 0.52 & 0.63 & 0.65 & 0.71 & 0.55 & 0.16 & 0.07 & 0.18 & 0.57 & 0.76 & 0.11 & 0.07 & 0.07 & 0.18 & 0.46 & 0.74 & 0.12 & 0.50 & 0.72 & 0.40 \\
\hline \multirow[t]{2}{*}{ grassland } & 31 & 0.51 & 0.71 & 0.77 & 0.79 & 0.83 & 0.48 & 0.77 & 0.89 & 0.74 & 0.80 & 0.85 & 0.52 & 0.74 & 0.89 & 0.79 & 0.86 & 0.89 & 0.80 & 0.85 & 0.90 \\
\hline & \multicolumn{21}{|c|}{ Accuracy by class } \\
\hline ild & 35 & 0.38 & 0.42 & 0.60 & 0.65 & 0.63 & 0.46 & 0.52 & 0.54 & 0.60 & 0.68 & 0.63 & 0.51 & 0.61 & 0.60 & 0.66 & 0.76 & 0.68 & 0.67 & 0.74 & 0.68 \\
\hline ad & 0.15 & 0.19 & 0.30 & 0.49 & 0.50 & 0.51 & 0.23 & 0.34 & 0.34 & 0.40 & 0.51 & 0.55 & 0.20 & 0.42 & 0.49 & 0.45 & 0.65 & 0.63 & 0.51 & 0.63 & 0.62 \\
\hline wat & 0.04 & 0.01 & 0.01 & 0.19 & 0.24 & 0.19 & 0.12 & 0.19 & 0.00 & 0.14 & 0.23 & 0.00 & 0.05 & 0.15 & 0.03 & 0.09 & 0.22 & 0.11 & 0.12 & 0.23 & 0.41 \\
\hline & & 0.41 & 0.41 & 0.35 & 0.44 & 0.63 & 0.13 & 0.11 & 0.26 & 0.48 & 0.39 & 0.37 & 0.19 & 0.12 & 0.39 & 0.43 & 0.36 & 0.36 & 0.31 & 0.38 & 0.70 \\
\hline grassland & 0.75 & 0.74 & 0.73 & 0.87 & 0.90 & 0.86 & 0.79 & 0.74 & 0.70 & 0.88 & 0.91 & 0.85 & 0.80 & 0.78 & 0.78 & 0.88 & 0.88 & 0.80 & 0.88 & 0.87 & 0.82 \\
\hline
\end{tabular}

Table 3. 


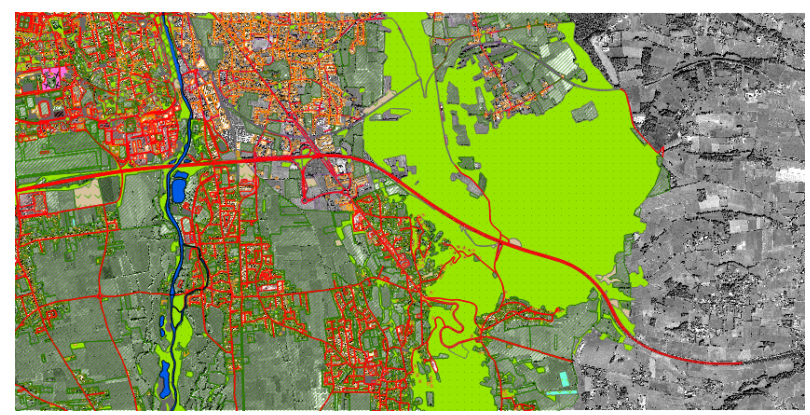

(a)

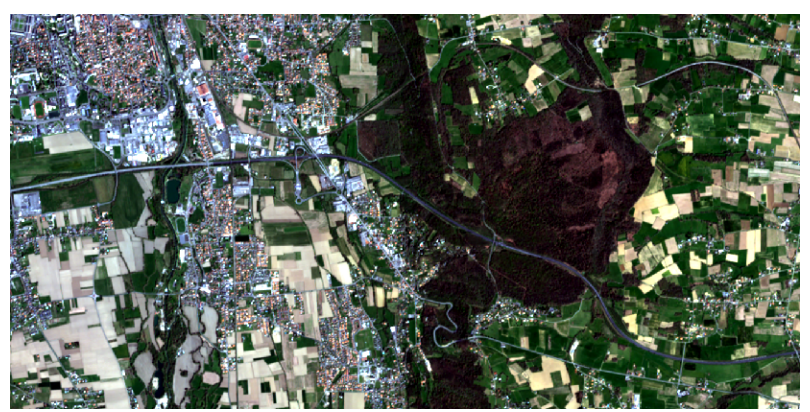

(b)

Fig. 1. (a) the French National Land Cover Database, and (b) an optical VHR image ( SPOT 6).

\section{CONCLUSION}

We proposed in this paper an adaptation of a generic landcover geodatabase updating framework, allowing to introduce multi-sensor data, regardless of sensor type. Three different fusion methods have been tested and compared with a comprehensive dataset composed of one Land-Cover geodatabases (LC-DB), and several remote sensing data (optical, SAR and LIDAR), acquired over the same area of interest. Preliminary results tend to show the complementarity of each individual sensor type (LIDAR for anthropic classes and optical for vegetation classes). Moreover, the 3 fusion processes have been successfully applied on one multi-temporal optical datasets (composed of 3 RapidEye images), showing the interest of tuning the fusion step. The extended version of this paper will contain the results of the 3 proposed fusion processes on various scenarii including optical, LIDAR and SAR datasets. Those scenarii will be assessed thanks to the existing LC-DB, and compared to a simple feature concatenation, scenario.

\section{REFERENCES}

[1] A. Belward and J. Skoien, "Who launched what, when and why; trends in global land-cover observation capacity from civilian earth observation satellites," ISPRS Journal of Photogrammetry and Remote Sensing, vol. in Press, Apr. 2014.

[2] C. Debes, A. Merentitis, R. Heremans, N. Frangiadakis, T. Kasteren, W. Liao, R. Bellens, S. Gautama, W. Philips, S. Prasad, Q. Du, and F. Pacifici, "Hyperspectral and LiDAR Data Fusion: Outcome of the 2013 GRSS Data Fusion Contest," IEEE Journal of Selected Topics in Applied Earth Observations and Remote Sensing, vol. 7, no. 6, pp. 2405-2418, 2014.
[3] A. Voisin, V. Krylov, G. Moser, S. Serpico, and J. Zerubia, "Supervised Classification of Multisensor and Multiresolution Remote Sensing Images With a Hierarchical Copula-Based Approach," IEEE Transactions on Geoscience and Remote Sensing, vol. 52, no. 6, pp. 33463358, 2014.

[4] M. Liu, M. Ozdogan, and X. Zhu, "Crop Type Classification by Simultaneous Use of Satellite Images of Different Resolutions," IEEE Transactions on Geoscience and Remote Sensing, vol. 52, no. 6, pp. 3637-3649, June 2014.

[5] A. Gressin, C. Mallet, N. Vincent, and N. Paparoditis, "Updating the new French national land cover database," in IEEE International Geoscience and Remote Sensing Symposium, Québec, Canada, 2014.

[6] L. Breiman, "Stacked Regressions," Machine Learning, vol. 24, pp. 49-64, 1996. 